\title{
Glucose affects cell viability, migration, angiogenesis and cellular adhesion of human retinal capillary endothelial cells via SPARC
}

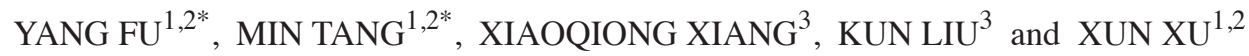 \\ ${ }^{1}$ Department of Ophthalmology, Shanghai General Hospital of Nanjing Medical University; ${ }^{2}$ Shanghai Key Laboratory of \\ Fundus Disease; ${ }^{3}$ Department of Ophthalmology, Shanghai General Hospital, Shanghai Jiao Tong University \\ School of Medicine, Shanghai 200080, P.R. China
}

Received March 15, 2018; Accepted September 6, 2018

DOI: $10.3892 /$ etm.2018.6970

\begin{abstract}
The expression of secreted protein acidic and rich in cysteine (SPARC) has been recently identified to be associated with the pathology of diabetic retinopathy. Therefore, the present study aimed to evaluate the regulatory role of SPARC in human retinal capillary endothelial cells (HRCECs), following exposure to a high glucose environment in vitro. The cell viability, migration, angiogenesis, permeability and SPARC expression levels of HRCECs were measured following treatment with different concentrations of glucose $(25,50$ or $100 \mathrm{mM})$. Lentiviral vectors (LV185-pL_shRNA_mKate2-SPARC-543; target sequence, GGATGAGGACAACAACCTTCT) that inhibit the expression of SPARC were constructed, and HRCECs were evaluated when infected by viruses carrying the lentiviral vectors. Cell viability was examined using the Cell Counting Kit- 8 assay. The expression of SPARC in HRCECs increased as the concentration of glucose in the culture medium increased. Relatively high concentrations of glucose significantly inhibited cell proliferation $(\mathrm{P}<0.05)$, migration $(\mathrm{P}<0.05)$, angiogenesis $(\mathrm{P}<0.01)$, and the expression of $\mathrm{ZO}$, occludin, claudin and JAM1 in tight junctions $(\mathrm{P}<0.01)$, gap junctions (Cx37 and $\mathrm{Cx} 43 ; \mathrm{P}<0.01)$ and adherens junctions (VE-cadherin, CTNNA1 and CTNNB1; P<0.05). However, when SPARC was downregulated by lentiviral vectors, the inhibitions induced by high concentrations of glucose were partially reversed. To conclude, the inhibitory effects on cell viability, migration, angiogenesis and cellular adhesion of HRCECs induced by high concentrations of glucose were reversed once the expression of SPARC was inhibited. These
\end{abstract}

Correspondence to: Dr Xun Xu, Department of Ophthalmology, Shanghai General Hospital of Nanjing Medical University, 100 Haining Road, Hongkou, Shanghai 200080, P.R. China

E-mail: xunxu170720@163.com

*Contributed equally

Key words: secreted protein acidic and rich in cysteine, human retinal capillary endothelial cells, diabetic retinopathy, SPARC findings suggest that SPARC may serve an important role in pathogenesis of diabetic retinopathy.

\section{Introduction}

Diabetic retinopathy is recognized as the most important manifestation of diabetic microvascular disease and the leading cause of vision loss, particularly among adults aged 20-74 years (1). Recently, the disease has become the fifth most common cause of severe visual impairment worldwide $(2,3)$. For patients with diabetic retinopathy, neovascularization is an important event involving various secretory proteins, including multiple growth factors, and angiogenesis has been recognized as the therapeutic target for diabetic retinopathy $(4,5)$.

Secreted protein acidic and rich in cysteine (SPARC), also known as osteonectin or BM-40, is a widely expressed profibrotic protein with pleiotropic roles that has been studied under a variety of conditions $(6,7)$. Previous results have demonstrated that SPARC may be a key component in the pathologies associated with obesity and diabetes (8). Furthermore, elevated plasma SPARC levels have been indicated to be associated with insulin resistance, dyslipidemia and inflammation in gestational diabetes mellitus (9). A previous study suggested that elevated plasma levels of SPARC were exhibited in patients with newly diagnosed type 2 diabetes mellitus (10). SPARC gene expression has also been revealed to be increased in diabetes-associated mesenteric vascular hypertrophy (11). However, the role of SPARC in glucose-mediated damage of endothelial cells remains unclear.

To study the role of SPARC in diabetic retinopathy, an ex vivo model of human retinal capillary endothelial cells (HRCECs) in the presence of high concentrations of glucose was established. The expression of SPARC in HRCECs was altered and cell viability, migration, angiogenesis and cellular adhesion were evaluated.

\section{Materials and methods}

Construction of lentiviral vectors. Viral vectors were constructed using 293T cells (cat. no. CRL-11268; American Type Culture Collection, Manassas, VA, USA). The 293A cells (cat. no. R70507; Invitrogen; Thermo Fisher Scientific, Inc., 
Waltham, MA, USA) were used for titration of lentiviral vectors by reverse transcription-quantitative-polymerase chain reaction (RT-qPCR). The 293T and 293A cell lines were cultured at $37^{\circ} \mathrm{C}$ in Opti-MEM (cat. no. 31985; Gibco; Thermo Fisher Scientific, Inc.) supplemented with $10 \%$ fetal bovine serum (cat. no. SH30084.03, Hyclone Laboratories, Inc., Logan, UT, USA). Lentiviruses were produced using a second-generation packaging system as described previously (12). Briefly, $6 \times 10^{6}$ 293T cells were plated in a $15-\mathrm{cm}$ dish and transfected with $9 \mu \mathrm{g}$ Packaging Mix (cat. no. K4975-00, Invitrogen; Thermo Fisher Scientific, Inc.), $3 \mu \mathrm{g}$ transfer plasmid and $36 \mu \mathrm{l}$ Lipofectamine 2000 (cat. no. 11668019, Invitrogen; Thermo Fisher Scientific, Inc.), which were combined in $5 \mathrm{ml}$ Opti-MEM. The transfection mix was removed once cells were cultured for $6 \mathrm{~h}$ at $37^{\circ} \mathrm{C}$, and fresh culture medium was added. Virus-containing medium was collected at $48 \mathrm{~h}$ post-transfection. Subsequently, the collected medium was filtered through a cellulose acetate membrane (pore size, $0.45 \mathrm{~mm}$ ).

The 293A cell lines were used to detect titration of lentiviral vectors. The $293 \mathrm{~A}$ cells were plated into each well of a 24-well plate and transfected using various quantities of lentivirus (1, 0.1 and $0.01 \mu \mathrm{l})$. Genomic DNA was extracted and the provirus titer was calculated using RT-qPCR at $72 \mathrm{~h}$ post-transfection. $\beta$-actin was used as the housekeeping gene. The target sequence for SPARC knockdown was as follows: 5'-GGATGAGGACAACAACCTTCT-3'.

Expression of SPARC in HRCECs. HRCECs (BeNa Culture Collection, Shanghai, China; http://www.bncc.org. cn/pro/p92/p_339792.html; primary cells, passage 4-5) were cultured in Dulbecco's modified Eagle's medium without glucose (cat. no. 11966025, Gibco; Thermo Fisher Scientific, Inc.) supplemented with $10 \%$ fetal bovine serum in a cell incubator with $5 \% \mathrm{CO}_{2}$ at $37^{\circ} \mathrm{C}$. The 3 rd to 5 th passaged cells were used for all experiments as described previously $(13,14)$. Different concentrations of glucose $(25,50$ or $100 \mathrm{mM})$ were added in culture medium, respectively. RT-qPCR was performed to evaluate SPARC expression in HRCECs treated with different concentrations of glucose. HRCECs were then infected with viruses carrying LV185-pL_shRNA_ mKate2-SPARC-543. HRCECs infected with empty vectors (negative control) or HRCECs that were not infected (blank control) served as controls. The expression of SPARC was assessed $48 \mathrm{~h}$ following this.

Cell viability assay. To determine the effects of SPARC on cell viability following treatment with different concentrations of glucose, HRCECs $\left(1 \times 10^{4}\right)$ were plated in a 96-well plate and infected with LV185-pL_shRNA_mKate2-SPARC-543 or empty vectors. Subsequently, $10 \mu 1$ of Cell Counting Kit- 8 (CCK-8) reagent (cat. no. CK04-05, Dojindo Molecular Technologies, Inc., Kumamoto, Japan) was added into each well for each of the three groups, respectively. The absorbance at $450 \mathrm{~nm}$ was measured to evaluate cell viability at 24,48 and $72 \mathrm{~h}$, respectively. Experiments were performed a total of three times.

Transwell migration assay. Cell migration was assessed by Transwell assay. Transwell plate was pretreated using Dulbecco's modified Eagle's medium (DMEM) without glucose (cat. no. 11966025; Thermo Fisher Scientific, Inc.) for $30 \mathrm{~min}$. HRCECs transfected with viruses carrying LV185-pL_shRNA_mKate2-SPARC-543, or negative control vectors were resuspended at a cell density of $1 \times 10^{5} \mathrm{cells} / \mathrm{ml}$ in DMEM without glucose. Cell suspension $(200 \mu \mathrm{l})$ was added to the upper chamber and $800 \mu \mathrm{l}$ culture medium with different concentrations of glucose $(25,50$ or $100 \mathrm{mM})$ was added to the lower chamber. Cells were allowed to migrate through the membrane for $24 \mathrm{~h}$. Following incubation, cells on top of the filter were scraped off using a cotton swab. The membrane was subsequently fixed in $4 \%$ paraformaldehyde for $10 \mathrm{~min}$ at room temperature, and stained with hematoxylin and eosin for $10 \mathrm{~min}$ at room temperature. The inserts were mounted on a slide, and the number of cells migrated to the bottom of filter was determined by counting 10 high-power fields with a bright field microscope (magnification, x200).

Measurement of angiogenesis. HRCECs were trypsinized and resuspended in extracellular matrix (ECM) culture media with $10 \%$ fetal bovine serum (Hyclone). Cells from the experimental groups were plated at a density of $1.5 \times 10^{4} \mathrm{cells} /$ well in triplicate in 96-well plates, respectively. Plates were incubated for $24 \mathrm{~h}$ at $37^{\circ} \mathrm{C}$ prior to photomicroscopy. Images from each well were taken at a magnification of $\mathrm{x} 4$ using an Olympus microscope (cat. no. CKX31; Olympus Corporation, Tokyo, Japan) every 2 h. Image-Pro Plus software (version 6.0.0.260; Media Cybernetics, Inc., Rockville, MD, USA) was utilized to calculate the length of blood vessels formed $(15,16)$. Statistical analysis was subsequently performed.

$R T-q P C R$. HRCECs were cultured in medium containing 25,50 or $100 \mathrm{mM}$ glucose, respectively. Following $24 \mathrm{~h}$, total RNA was extracted from HRCECs. The expression levels of the following genes were detected using RT-qPCR: Genes associated with tight junctions, including zonula occludens (ZO)-1, occludin, claudin and junctional adhesion molecule 1 (JAM1); genes associated with gap junctions, including connexin (Cx)37, Cx40 and $\mathrm{Cx} 43$; genes associated with adherens junctions, including vascular endothelial (VE)-cadherin, catenin alpha 1 (CTNNA1), catenin beta 1 (CTNNB1) and catenin delta 1 (CTNND1). Total RNA was extracted using TRIzol (cat. no. 15596018; Invitrogen; Thermo Fisher Scientific, Inc.) according to the manufacturer's instructions. A cDNA synthesis kit (SuperScript First-Strand Synthesis System; cat. no. 11904-018; Invitrogen; Thermo Fisher Scientific, Inc.) was used for RT. Each reaction contained $0.5 \mu \mathrm{l}$ of random primers $(0.2 \mu \mathrm{g} / \mu \mathrm{l})$ and $1 \mu \mathrm{l}$ of SuperScript III reverse transcriptase $(200 \mathrm{U} / \mu \mathrm{l})$. The specific primers used are listed in Table I. qPCR was performed by utilizing the SYBR ${ }^{\circledR}$ Premix Ex Taq (cat. no. RR420L; Takara Bio, Inc., Otsu, Japan). The following PCR conditions were used: Pre-denaturing at $95^{\circ} \mathrm{C}$ for $2 \mathrm{~min}$; denaturing at $95^{\circ} \mathrm{C}$ for $10 \mathrm{sec}$; and annealing and polymerization at $60^{\circ} \mathrm{C}$ for $30 \mathrm{sec}$ and $70^{\circ} \mathrm{C}$ for $45 \mathrm{sec}$. A total of 40 PCR cycles were performed. PCR was performed using a CFX96 Touch Real-Time PCR Detection System. Gene expression was determined as the ratio of relative optical density of target gene to $\beta$-actin. The $2^{-\Delta \Delta \mathrm{Cq}}$ method was utilized to measure PCR results (17). 
Table I. Primers used in reverse transcription-quantitative polymerase chain reaction.

\begin{tabular}{|c|c|c|}
\hline Genes & Primers & Sequences $\left(5^{\prime}\right.$ to $\left.3^{\prime}\right)$ \\
\hline \multirow[t]{2}{*}{ SPARC } & SPARC-F & AGGAAACCGAAGAGGAGG \\
\hline & SPARC-R & GCAAAGAAGTGGCAGGAA \\
\hline \multirow[t]{2}{*}{$\mathrm{ZO}-1$} & $\mathrm{ZO}-1-\mathrm{F}$ & TATTCACGCAGTTACGAGCAAG \\
\hline & $\mathrm{ZO}-1-\mathrm{R}$ & AAGGTATCAGCGGAGGGACA \\
\hline \multirow[t]{2}{*}{ Occludin } & Occludin-F & AAGCCAAACCTCTGTGAGCA \\
\hline & Occludin-R & GGAGTGTTCAGCCCAGTTGA \\
\hline \multirow[t]{2}{*}{ Claudin } & Claudin-F & TTGGGCTTCATTCTCGCCTT \\
\hline & Claudin-R & GAGGATGCCAACCACCATCA \\
\hline \multirow[t]{2}{*}{ JAM1 } & JAM1-F & AATCTGCACTCAACTGCCCA \\
\hline & JAM1-R & CATTTCAGCCGCTCTAGCCT \\
\hline \multirow[t]{2}{*}{ Cx37 } & Cx37-F & ACCATGCCCCACCTACAATG \\
\hline & Cx37-R & CAGCCAGACTTCTCAGGACC \\
\hline \multirow[t]{2}{*}{$\mathrm{Cx} 40$} & $\mathrm{Cx} 40-\mathrm{F}$ & GCATTGTGCTTCTCGGGTTC \\
\hline & $\mathrm{Cx} 40-\mathrm{R}$ & CTATCACCACACCTCTCGGC \\
\hline \multirow[t]{2}{*}{$\mathrm{Cx} 43$} & $\mathrm{Cx} 43-\mathrm{F}$ & TTCAAGGGCGTTAAGGATCG \\
\hline & $\mathrm{Cx} 43-\mathrm{R}$ & TAACCAGCTTGTACCCAGGA \\
\hline \multirow[t]{2}{*}{ VE-cadherin } & VE-cadherin-F & AAGCAGAGCTGGGTCACTTG \\
\hline & VE-cadherin-R & GAAGCTCTTGGCTCTGGAGG \\
\hline \multirow[t]{2}{*}{ CTNNA1 } & CTNNA1-F & TAACAGCATCGGGGTCGTTG \\
\hline & CTNNA1-R & GGCTGGTGTTAAATCAGCACT \\
\hline \multirow[t]{2}{*}{ CTNNB1 } & CTNNB1-F & GTCTGAGGACAAGCCACAAGA \\
\hline & CTNNB1-R & GCCACCCATCTCATGTTCCA \\
\hline \multirow[t]{2}{*}{ CTNND1 } & CTNND1-F & AGCTGGCTGCCTTAGGAATG \\
\hline & CTNND1-R & ATCTTTGCCCTTCTTGGCCC \\
\hline
\end{tabular}

SPARC, secreted protein acidic and rich in cysteine; ZO, zonula occludens; JAM1, junctional adhesion molecule 1; Cx, connexin; VE, vascular endothelial; CTNNA1, catenin alpha 1; CTNNB1, catenin beta 1; CTNND1, catenin delta 1; F, forward; R, reverse.

Western blot analysis. Protein expression levels of SPARC in HRCECs were detected using western blot analysis. HRCECs were lysed in lysis buffer (cat. no. P0013, Beyotime Institute of Biotechnology, Haimen, China) at $4^{\circ} \mathrm{C}$ with inhibitors of phosphatase and protease (cat. no. P1045, Beyotime Institute of Biotechnology). The lysis mixture was centrifuged at $4^{\circ} \mathrm{C}$ for $10 \mathrm{~min}$ at $10,000 \mathrm{x} \mathrm{g}$, and the supernatant containing cellular proteins was utilized in the following experiments. The protein concentration was measured using a BCA kit. Proteins were separated using SDS-PAGE (10\% gels, $40 \mu \mathrm{g}$ per lane; $120 \mathrm{~V})$. The separated proteins were then transferred to polyvinylidene fluoride membranes (100 V for $120 \mathrm{~min}$; cat. no. FFP24, Beyotime Institute of Biotechnology). The membranes were blocked with $5 \%$ non-fat milk at room temperature for $1 \mathrm{~h}$ and subsequently incubated with anti-SPARC (cat. no. ab225716; Abcam; $1: 1,000$ ) and anti- $\beta$-actin (cat. no. ab8227; Abcam; 1:1,000) primary antibodies against SPARC at $4^{\circ} \mathrm{C}$ overnight. Membranes were washed with Tris-buffered saline containing Tween 20 and incubated with horseradish peroxidase-conjugated goat anti-rabbit secondary antibody (cat. no. ab6721; Abcam; 1:2,000) at room temperature for $1 \mathrm{~h}$. Membranes were incubated in enhanced chemiluminescence solution (cat. no. P0018A, Beyotime Institute of Biotechnology). Images were captured on film (cat. no. FF057, Beyotime Institute of
Biotechnology) in a dark room. Experiments were repeated for 3 times. Blot images were quantified in greyscale using ImageJ (version 1.5.2; National Institute of Health, Bethesda, MD, USA).

Statistical analyses. Data were analysed using GraphPad Prism 5.0 software (GraphPad Software Inc., La Jolla, CA, USA). Data were presented as mean \pm standard deviation. Differences among three groups were measured by analysis of variance followed by the Bonferroni's post-hoc test for multiple comparisons. Differences between two groups were measured using a Student's t-test. $\mathrm{P}<0.05$ was considered to indicate a statistically significant difference.

\section{Results}

Treatment with glucose increases SPARC expression levels in HRCECs. The mRNA expression levels of SPARC in HRCECs was detected by RT-qPCR at 24, 48 and 72 h, respectively. Compared with the $25 \mathrm{mM}$ glucose group, the mRNA expression levels of SPARC in the $100 \mathrm{mM}$ glucose group were significantly increased at 24, 48 and $72 \mathrm{~h}(\mathrm{P}<0.05$ and $\mathrm{P}<0.01$; Fig. 1A). In addition, protein expression levels of SPARC in HRCECs were detected by western blot analysis at $72 \mathrm{~h}$. Compared with the 

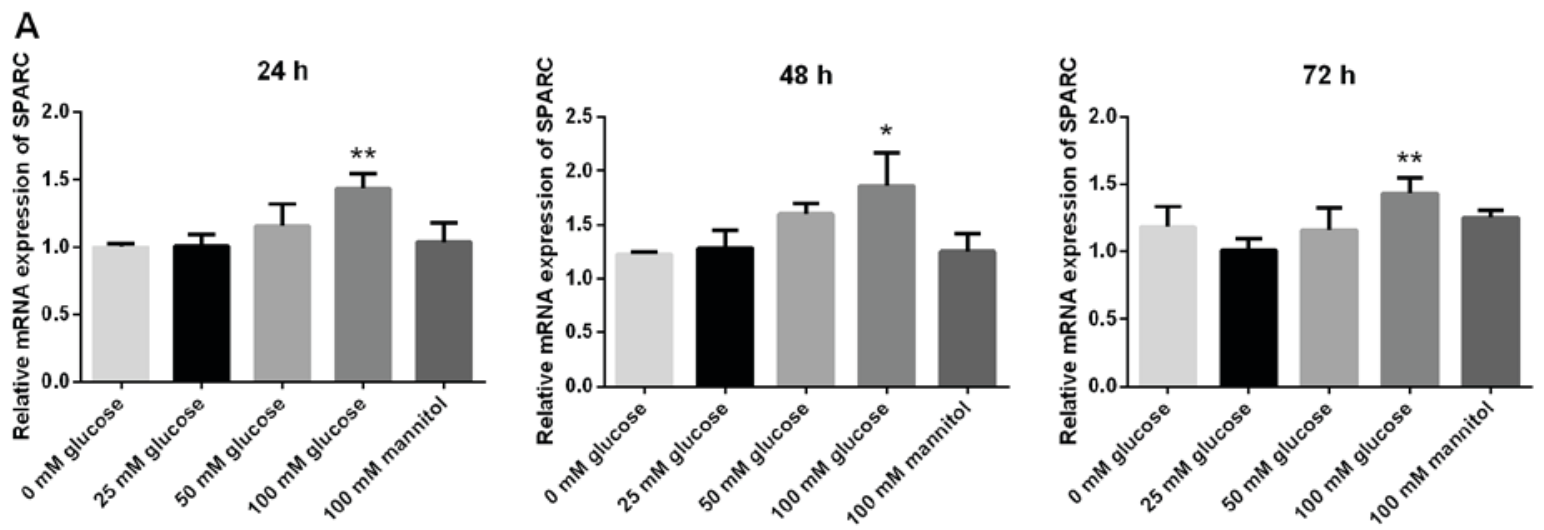

B

12345
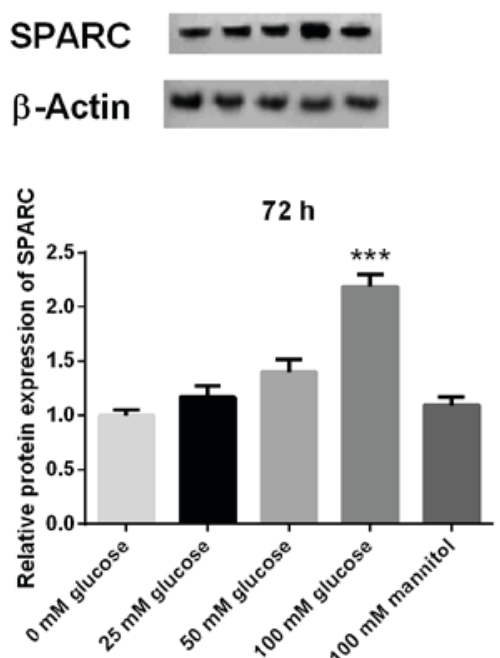

Figure 1. Expression of SPARC in HRCECs treated with $100 \mathrm{mM}$ glucose is significantly increased. (A) mRNA and (B) protein expression levels of SPARC The mRNA expression levels of SPARC in HRCECs was detected by reverse transcription-quantitative polymerase chain reaction at 24, 48, and $72 \mathrm{~h}$, respectively. Western blot analysis was used to detect the protein expression levels of SPARC. Data were presented as the mean \pm standard deviation ( $\mathrm{n}=3$ ). ${ }^{*} \mathrm{P}<0.05$, ${ }^{* *} \mathrm{P}<0.01$ and ${ }^{* * *} \mathrm{P}<0.001$ vs. $25 \mathrm{mM}$ glucose group. SPARC, secreted protein acidic and rich in cysteine; HRCECs, human retinal capillary endothelial cells; 1, blank; 2, 25 mM glucose; 3, 50 mM glucose; 4, 100 mM glucose; 5, $100 \mathrm{mM}$ mannitol.

$25 \mathrm{mM}$ glucose group, the protein expression levels of SPARC in the $100 \mathrm{mM}$ glucose group were significantly increased at $72 \mathrm{~h}(\mathrm{P}<0.001$; Fig. 1B). HRCECs without treatment (blank group) and $100 \mathrm{mM}$ mannitol served as controls.

SPARC interference increases the viability of HRCECs treated with glucose. The success of SPARC interference was confirmed by RT-qPCR. The expression of SPARC in the SPARC interference group was significantly decreased compared with blank or negative control groups $(\mathrm{P}<0.001$; Fig. 2). The interference efficiency was $89.34 \%$ (Fig. 2). Cell viability was detected using the CCK-8 assay. The SPARC interference group exhibited significantly increased cell viability when compared with the blank or negative control groups at 24, 48 and $72 \mathrm{~h}$ (all $\mathrm{P}<0.05$; Fig. 3).

SPARC interference enhances the migration of HRCECs treated with glucose. The migration ability of HRCECs was detected using the Transwell assay. The SPARC interference group exhibited significantly increased cell migration following treatment with 25,50 and $100 \mathrm{mM}$ glucose respectively, compared with the negative control group $(\mathrm{P}<0.05$;

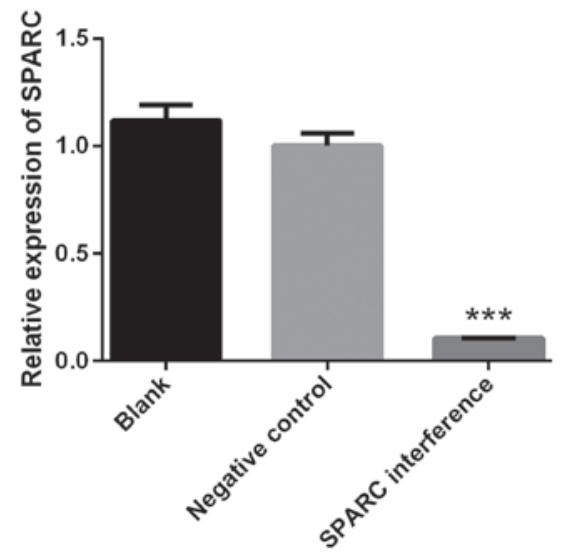

Figure 2. Confirmation of SPARC interference. The success of SPARC interference was confirmed by reverse transcription-quantitative polymerase chain reaction. Data were presented as the mean \pm standard deviation $(n=3)$. ${ }^{* * * *} \mathrm{P}<0.001$ vs. blank or negative control group. SPARC, secreted protein acidic and rich in cysteine.

Fig. 4). Results indicated that the migration ability was higher when the concentration of glucose was lower. 
$24 \mathrm{~h}$

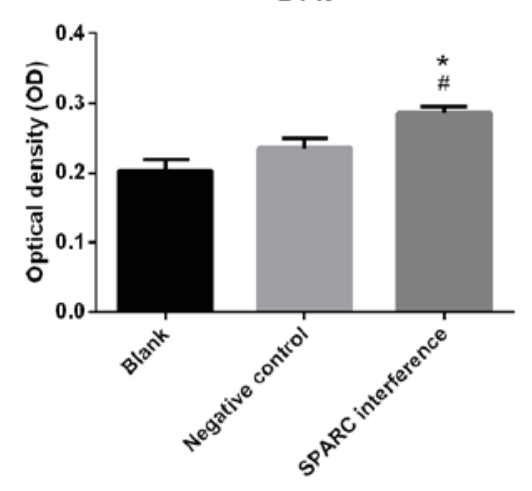

$48 \mathrm{~h}$

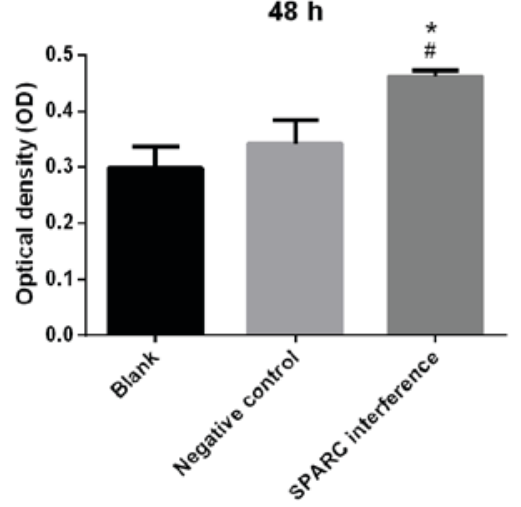

$72 \mathrm{~h}$

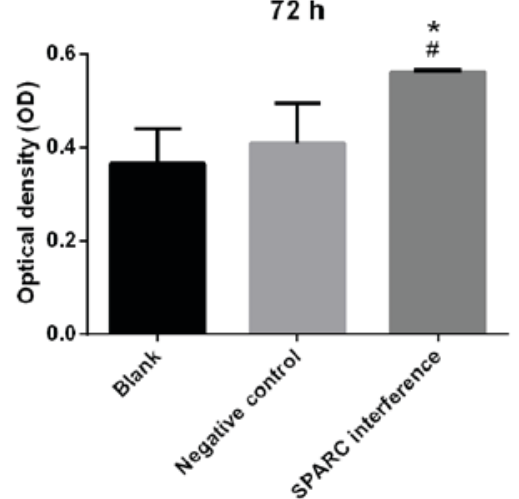

Figure 3. SPARC interference group increases the viability of human retinal capillary endothelial cells. Cell viability was detected using the Cell Counting Kit- 8 assay. Data were presented as the mean \pm standard deviation $(n=3)$. ${ }^{*} \mathrm{P}<0.05$ vs. blank group; ${ }^{\prime} \mathrm{P}<0.05$ vs. the negative control group. SPARC, secreted protein acidic and rich in cysteine.

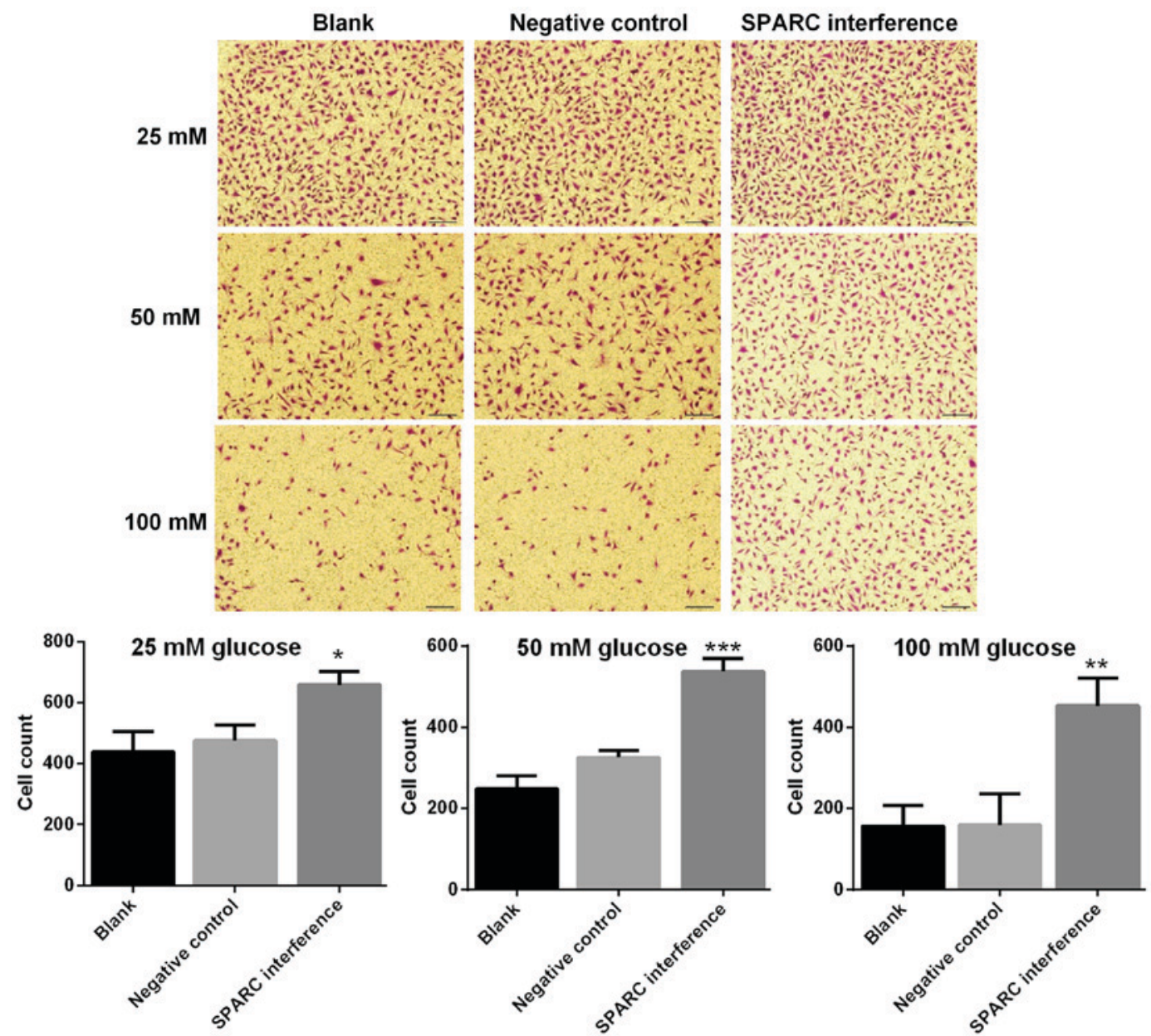

Negative control SPARC interference

Figure 4. SPARC interference increases the migration ability of HRCECs treated with glucose. HRCECs were incubated with glucose for $24 \mathrm{~h}$. Cell migration ability of HRCECs was detected using the Transwell assay (magnification, $\mathrm{x} 100)$. Data were presented as mean \pm standard deviation $(\mathrm{n}=3)$. ${ }^{*} \mathrm{P}<0.05$, ** $\mathrm{P}<0.01$, and ${ }^{* * *} \mathrm{P}<0.001$ vs. negative control group. SPARC, secreted protein acidic and rich in cysteine; HRCECs, human retinal capillary endothelial cells.

SPARC interference increases the length of blood vessels treated with glucose. Image-Pro Plus software was utilized to calculate the length of blood vessels formed with HRCECs. Compared with the negative control group, the SPARC interference group exhibited significantly increased vascular length following treatment with 25,50 and $100 \mathrm{mM}$ glucose $(\mathrm{P}<0.01$ and $\mathrm{P}<0.001$; Fig. 5).
SPARC interference increases the expression of genes associated with tight junctions, gap junctions and adherens junctions. The expression levels of genes associated with tight junctions (ZO-1, occludin, claudin and JAM1), gap junctions (Cx37, Cx40 and $\mathrm{Cx} 43$ ), and adherens junctions (VE-cadherin, CTNNA1, CTNNB1 and CTNND1) were detected using RT-qPCR. The gene expression levels of SPARC in the 

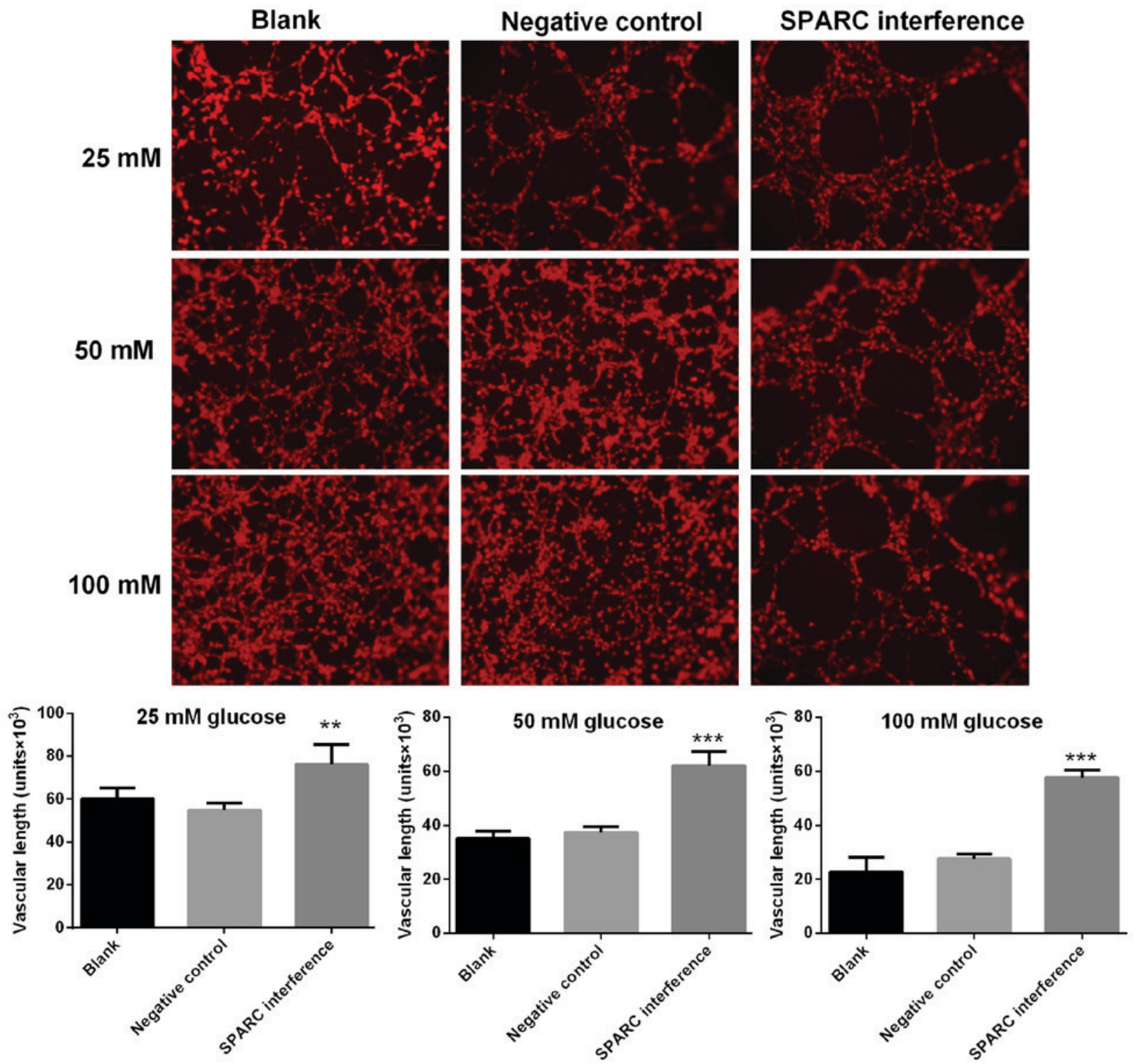

Figure 5. SPARC interference increases the vascular length of HRCECs treated with glucose. HRCECs were incubated with glucose for $24 \mathrm{~h}$. Image-Pro Plus software was utilized to calculate the length of blood vessels formed. Data were presented as mean \pm standard deviation $(\mathrm{n}=3) .{ }^{* * *} \mathrm{P}<0.01$ and ${ }^{* * * *} \mathrm{P}<0.001 \mathrm{vs}$. negative control group. SPARC, secreted protein acidic and rich in cysteine; HRCECs, human retinal capillary endothelial cells.

SPARC interference group that received treatment with 25, 50 and $100 \mathrm{mM}$ glucose were significantly decreased compared with the negative control group $(\mathrm{P}<0.01$ and $\mathrm{P}<0.001$; Fig. 6). Furthermore, following treatment with 25, 50 and $100 \mathrm{mM}$ glucose, the SPARC interference group demonstrated significantly increased expression levels of genes associated with tight junctions ( $\mathrm{ZO}$, occludin, claudin and JAM1; $\mathrm{P}<0.01$ and $\mathrm{P}<0.001$; Fig. 7). In addition, following treatment with glucose, the SPARC interference group exhibited increased expression levels of genes associated with gap junctions when compared with the negative control group. These differences were indicated to be significant with regard to $\mathrm{Cx} 37$ in the presence of 25, 50 and $100 \mathrm{mM}$ glucose and $\mathrm{Cx} 43$ in the presence of 25, 50 and $100 \mathrm{mM}(\mathrm{P}<0.01$ and $\mathrm{P}<0.001$; Fig. 8). Furthermore, following treatment with glucose, the SPARC interference group displayed significantly increased expression levels of genes associated with adherens junctions compared with the negative control group (VE-cadherin and CTNNA1, significant in the presence of 50 and $100 \mathrm{mM}$ glucose; CTNNB1 and CTNND1, significant in the presence of 25, 50 and $100 \mathrm{mM}$ glucose; $\mathrm{P}<0.05, \mathrm{P}<0.01$ and $\mathrm{P}<0.001$; Fig. 9).

\section{Discussion}

The present study demonstrated that the cell viability, migration, cellular adhesion, and angiogenesis were significantly inhibited by high concentrations of glucose, and the inhibition could be reversed once the expression of SPARC was inhibited.

SPARC is a widely expressed profibrotic protein with pleiotropic roles, which have been studied in a variety of conditions $(18,19)$. Previous evidence has demonstrated that SPARC was associated with human obesity and diabetes (11). In the present study, HRCECs were cultured in vitro and a stably transfected SPARC interference cell line was established. Notably, culture medium may contain glucose (20). However, the same volume and type of culture medium was added into 

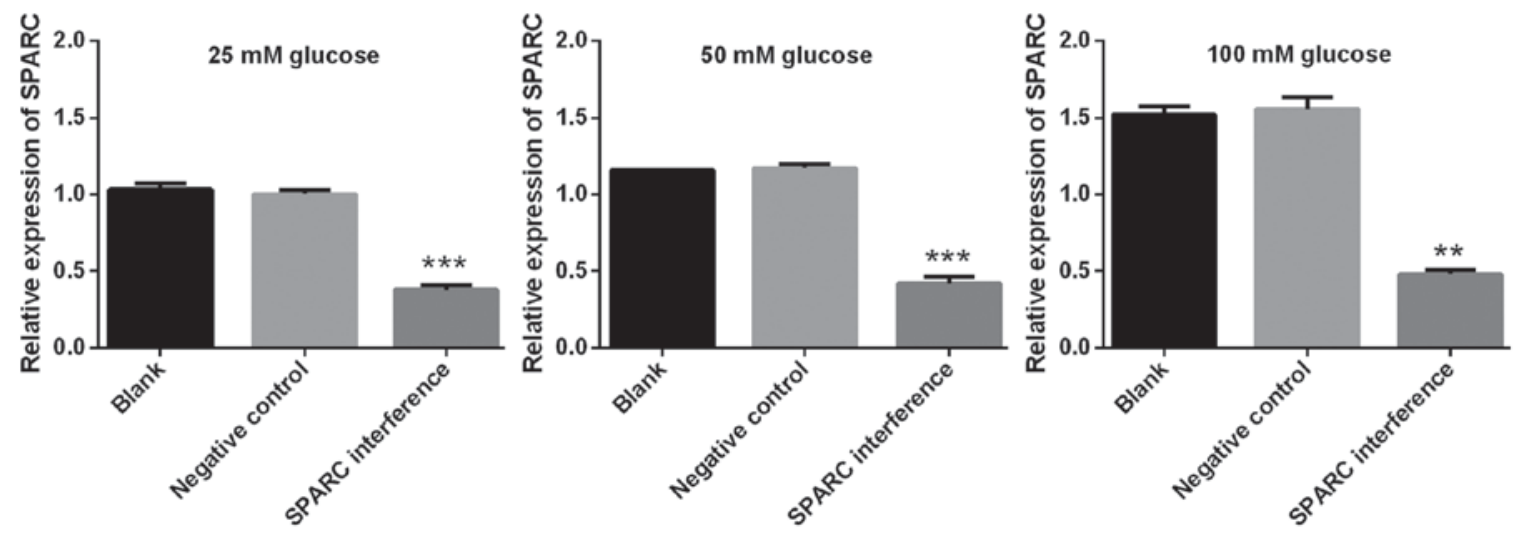

Figure 6. Expression of SPARC in HRCECs treated with glucose. HRCECs were incubated with glucose for $24 \mathrm{~h}$. Data were presented as mean \pm standard deviation $(\mathrm{n}=3) .{ }^{* *} \mathrm{P}<0.01$ and ${ }^{* * * *} \mathrm{P}<0.001$ vs. negative control group. SPARC,secreted protein acidic and rich in cysteine; HRCECs, human retinal capillary endothelial cells.

blank, negative control and SPARC groups. Therefore, the presence of any glucose in culture medium did not alter the comparisons among three experimental groups.

SPARC is a modulator of cell-surface interaction (21). Tissue physiology can be modulated by SPARC by altering cell proliferation and migration, and interactions between cell and ECM (22). A previous study revealed that adipocytes were recognized as the major source for producing circulating SPARC, and insulin resistance in adipose tissue may be caused by increased SPARC expression (23). Notably, the role of SPARC in diabetic retinopathy has been rarely studied. However, the role of SPARC in diabetes has been extensively studied. Xu et al (9) and Wu et al (10) have demonstrated that SPARC is an independent negative indicator of diabetes, which correlates significantly with inflammation, dyslipidemia and insulin resistance. In the present study, the expression of SPARC was significantly increased when HRCECs were stimulated with high concentrations of glucose. Watanabe et al (24) demonstrated that the expression of SPARC was high in proliferative diabetic retinopathy using oligo-cap signal sequence trap strategy. These findings indicate that SPARC may serve a potential regulatory role in patients with diabetic retinopathy, which is a major complication among patients with diabetes (25).

The present study revealed that high concentrations of glucose inhibited cell viability and migration, which are essential for the turnover and regeneration of capillary endothelial cells (26). HRCECs may be vulnerable to damage induced by pathological processes of diabetic retinopathy, including oxidative stress, inflammation, ischemia-reperfusion and hypoxia (27). Furthermore, impaired endothelial cells, along with other vascular dysfunctions, can act as an important implication for early diabetic retinopathy (28). The present study suggested that downregulation of SPARC increased cell viability and migration, which may have protective effects on capillary endothelial cells in diabetic retinopathy.

The present data revealed that SPARC was highly expressed, and angiogenesis was inhibited, when HRCECs were cultured with high concentrations of glucose. Furthermore, when the expression of SPARC was inhibited, angiogenesis was recovered. Previous data demonstrated that SPARC was cell-type specific in angiogenesis (29).
Until now, to the best of our knowledge no direct evidence has revealed that SPARC is associated with angiogenesis in retinopathy. In gastric cancer, Zhang et al (30) demonstrated that the suppression of angiogenesis by SPARC may be mediated by downregulating the expression of vascular endothelial growth factor (VEGF) and matrix metalloproteinases (MMP-7). Notably, overexpression of SPARC has also been demonstrated to be involved in radiation-reduced tumor angiogenesis by downregulating VEGF-A via microRNA-410 (31). The pathway that is associated with angiogenesis in retinopathy and is regulated by SPARC remains unclear. However, the present data suggested that the cell viability, migration and permeability was significantly associated with SPARC expression. Further studies are required to fully explore the possible molecular mechanisms of SPARC in angiogenesis in patients with diabetic retinopathy.

The present findings also indicated that high concentrations of glucose significantly inhibited the expression of genes associated with cell tight junctions, gap junctions and adherens junctions. However, when SPARC was downregulated, the expression levels of those genes increased. Previous research has demonstrated that the collapse of cell junctions caused hyperpermeability of retinal capillary endothelial cells and vascular leakage (32). In patients with diabetic retinopathy, such hyperpermeability caused leakage of macromolecules, proteins and electrolytes into tissue space with sera, thereby increasing interstitial osmolarity and exacerbating vascular leakage. This in turn formed severe diabetic macular edema (DME) (33). Nowadays, DME is known as one of most severe complications and leading cause of vision loss in diabetic retinopathy (34). SPARC, as a key regulator in cell permeability, may be promising in treatment of DME in future clinical practice.

In conclusion, the present data demonstrated that cell viability, migration, cellular adhesion and angiogenesis of retinal capillary endothelial cells exposed to high concentrations of glucose may be mediated, at least in part, by changes in the expression of SPARC. Alterations of SPARC expression may contribute to the development and pathogenesis of diabetic retinopathy. Although further studies are required to fully clarify the role of SPARC in neovascularization and 

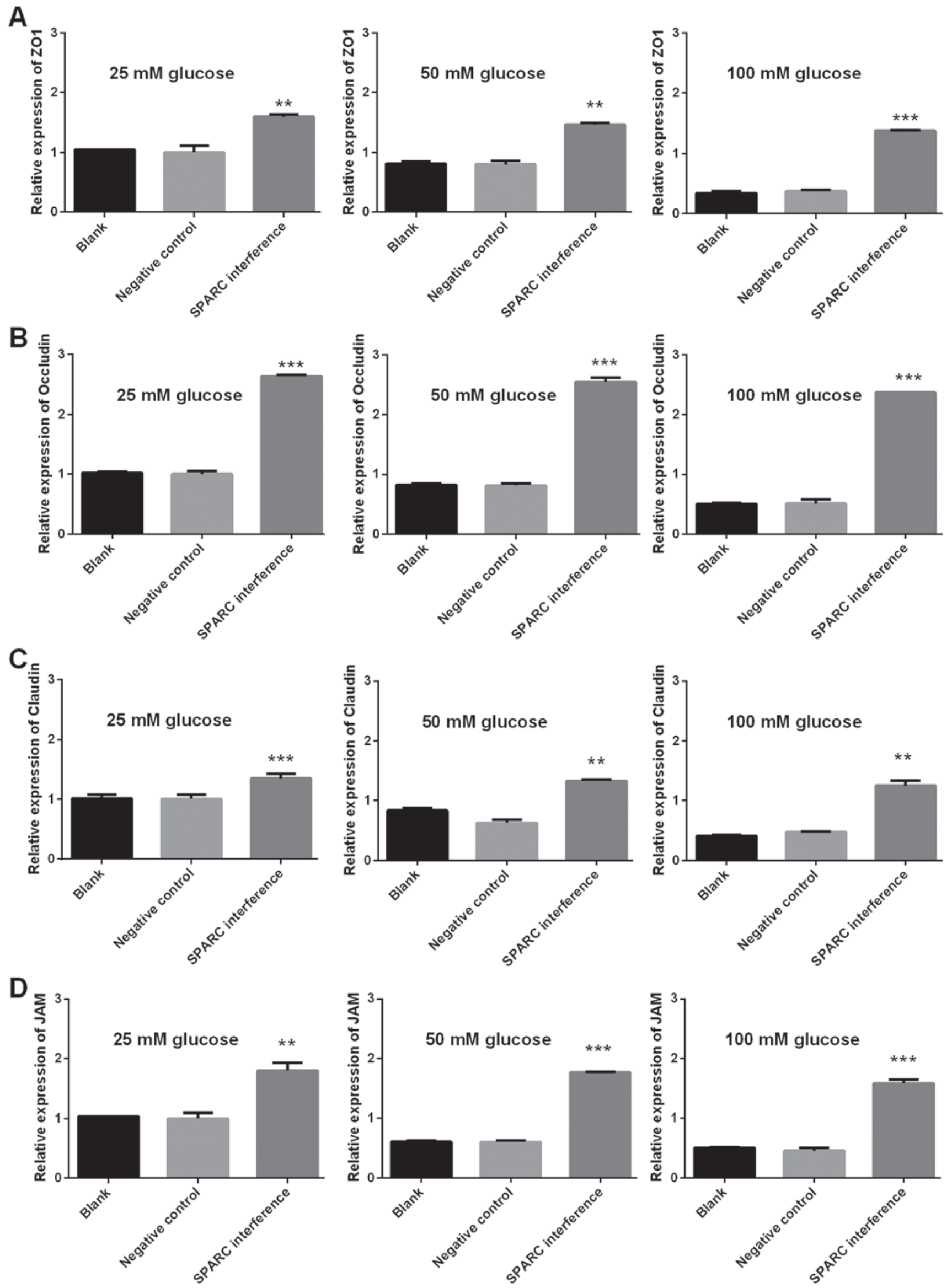

Figure 7. SPARC interference increases the expression of tight junction-associated expression of genes in HRCECs treated with glucose. (A) Expression of ZO1, (B) occluding, (C) claudin and (D) JAM1. HRCECs were incubated with glucose for $24 \mathrm{~h}$. Gene expression was detected using reverse transcription-quantitative polymerase chain reaction. Data were presented as mean \pm standard deviation $(\mathrm{n}=3)$. ${ }^{* *} \mathrm{P}<0.01$ and ${ }^{* * * *} \mathrm{P}<0.001$ vs. negative control group. SPARC, secreted protein acidic and rich in cysteine; HRCECs, human retinal capillary endothelial cells; JAM, junctional adhesion molecule; ZO-1, zonula occludens. 

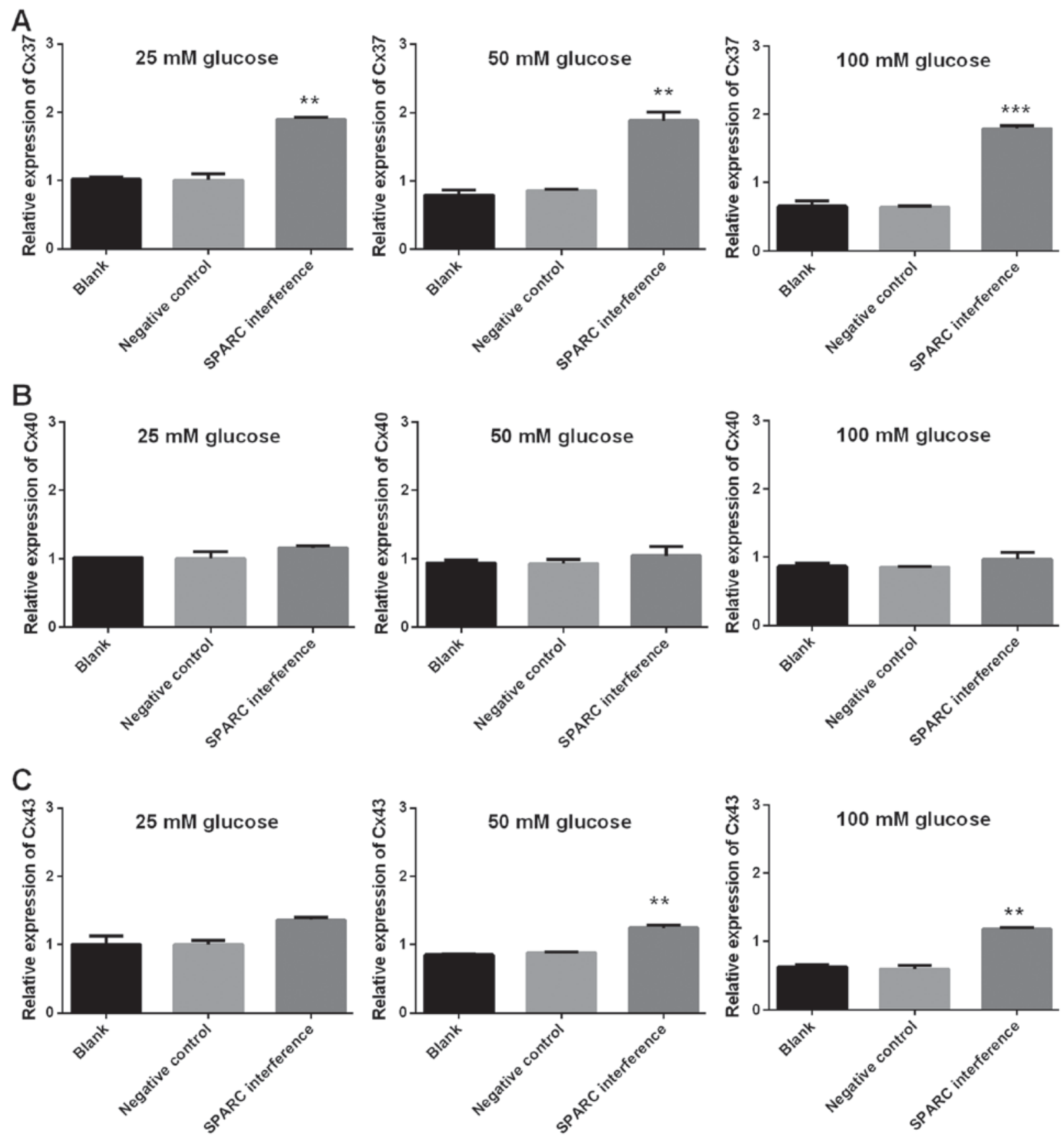

Figure 8. SPARC interference increases the expression of gap junction-associated genes associated in HRCECs treated with glucose. (A) Expression of Cx37, (B) Cx40 and (C) Cx43. HRCECs were incubated with glucose for $24 \mathrm{~h}$. Gene expression was examined using reverse transcription-quantitative polymerase chain reaction. Data were presented as mean \pm standard deviation $(n=3) .{ }^{* *} \mathrm{P}<0.01$ and ${ }^{* * *} \mathrm{P}<0.001$ vs. negative control group. SPARC, secreted protein acidic and rich in cysteine; HRCECs, human retinal capillary endothelial cells; $\mathrm{Cx}$, connexin.

fibrosis, the present analysis on the role of SPARC expression may provide insights on future therapeutic approaches for diabetic retinopathy.

\section{Acknowledgements}

Not applicable.

\section{Funding}

The present study was supported by the National Natural Science Fund of China (grant no. 81570851).

\section{Availability of data and materials}

The datasets used and/or analyzed during the current study are available from the corresponding author on reasonable request.

\section{Authors' contributions}

XX designed and performed the experiments. YF and MT interpreted the data, drafted the manuscript and provided experimental support. XQX and KL collected and analyzed the data. All authors read and approved the final manuscript. 
$\mathrm{A}$ 든
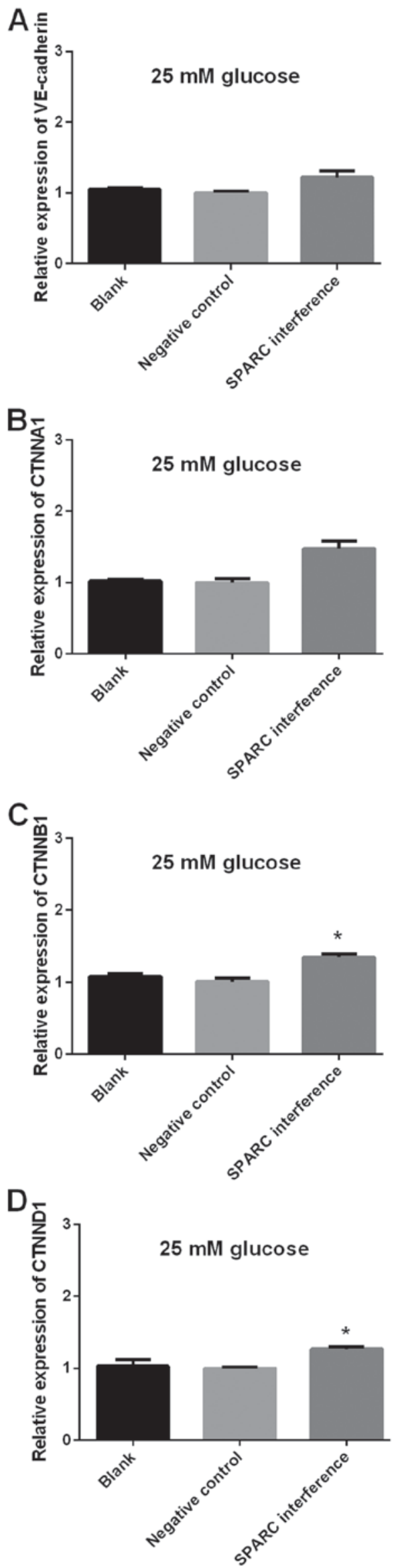
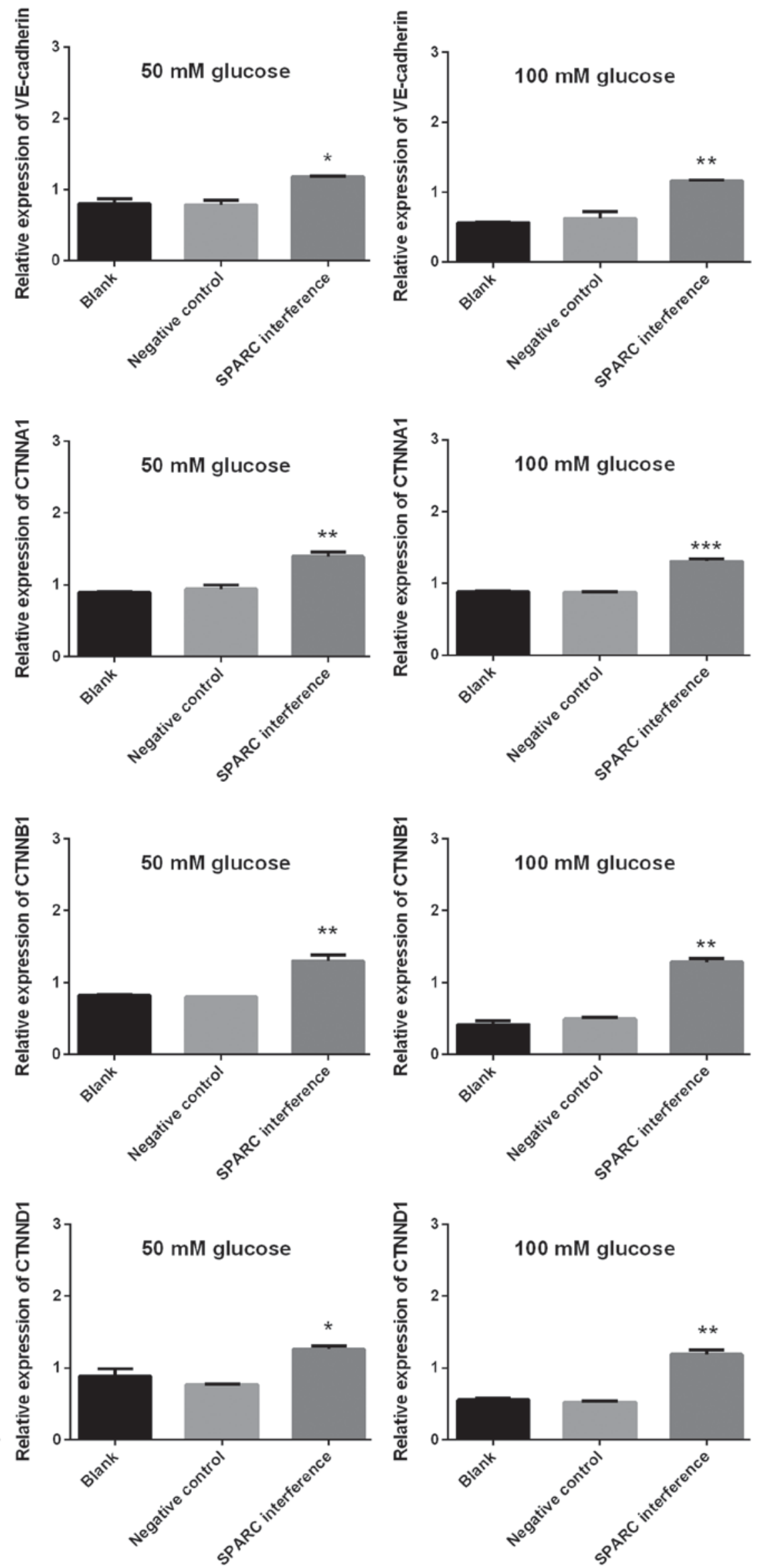

Figure 9. SPARC interference increases the expression of genes associated with adherens junctions in HRCECs treated with glucose. (A) Expression of VE-cadherin, (B) CTNNA1, (C) CTNNB1 and (D) CTNND1. HRCECs were incubated with glucose for $24 \mathrm{~h}$. Data were presented as mean \pm standard deviation $(\mathrm{n}=3)$. ${ }^{*} \mathrm{P}<0.05,{ }^{* *} \mathrm{P}<0.01$ and ${ }^{* * *} \mathrm{P}<0.001$ vs. negative control group. SPARC, secreted protein acidic and rich in cysteine; HRCECs, human retinal capillary endothelial cells; VE, vascular endothelial; CTNNA1, catenin alpha 1; CTNNB1; catenin beta 1 CTNND1, catenin delta 1. 


\section{Ethics approval and consent to participate}

The present study was approved by the Ethics Committee of Shanghai General Hospital, (Shanghai, China; Registration no. 2015 K061).

\section{Patient consent for publication}

Not applicable.

\section{Competing interests}

The authors declare that they have no competing interests.

\section{References}

1. Smith-Morris C, Bresnick GH, Cuadros J, Bouskill KE and Pedersen ER: Diabetic retinopathy and the cascade into vision loss. Med Anthropol 17: 1-14, 2018.

2. Gella L, Raman R, Pal SS, Ganesan S and Sharma T: Incidence, progression, and associated risk factors of posterior vitreous detachment in type 2 diabetes mellitus: Sankara nethralaya diabetic retinopathy epidemiology and molecular genetic study (SN-DREAMS II, Report No. 7). Semin Ophthalmol 32: 191-197, 2017.

3. Raman R, Ganesan S, Pal SS, Gella L, Kulothungan V and Sharma T: Incidence and progression of diabetic retinopathy in urban India: Sankara nethralaya-diabetic retinopathy epidemiology and molecular genetics study (SN-DREAMS II), Report 1. Ophthalmic Epidemiol 24: 294-302, 2017.

4. Capitão M and Soares R: Angiogenesis and inflammation crosstalk in diabetic retinopathy. J Cell Biochem 117: 2443-2453, 2016

5. Zhou L, Zhang T, Lu B, Yu Z, Mei X, Abulizi P and Ji L: Lonicerae Japonicae Flos attenuates diabetic retinopathy by inhibiting retinal angiogenesis. J Ethnopharmacol 189: 117-125, 2016.

6. Tanaka M, Takagi T, Naito Y, Uchiyama K, Hotta Y, Toyokawa Y, Ushiroda C, Hirai Y, Aoi W, Higashimura Y, et al: Secreted protein acidic and rich in cysteine functions in colitis via IL17A regulation in mucosal CD4+ T cells. J Gastroenterol Hepatol 33 671-680, 2018

7. Toyota K, Murakami Y, Kondo N, Uemura K, Nakagawa N, Takahashi $S$ and Sueda T: Impact of secreted protein acidic and rich in cysteine (SPARC) expression on prognosis after surgical resection for biliary carcinoma. J Gastrointest Surg 21: 990-999, 2017.

8. Kos K and Wilding JP: SPARC: A key player in the pathologies associated with obesity and diabetes. Nat Rev Endocrinol 6: 225-235, 2010.

9. Xu L, Ping F, Yin J, Xiao X, Xiang H, Ballantyne CM, Wu H and Li M: Elevated plasma SPARC levels are associated with insulin resistance, dyslipidemia, and inflammation in gestational diabetes mellitus. PLoS One 8: e81615, 2013.

10. Wu D, Li L, Yang M, Liu H and Yang G: Elevated plasma levels of SPARC in patients with newly diagnosed type 2 diabetes mellitus. Eur J Endocrinol 165: 597-601, 2011.

11. Jandeleit-Dahm K, Rumble J, Cox AJ, Kelly DJ, Dziadek M, Cooper ME and Gilbert RE: SPARC gene expression is increased in diabetes-related mesenteric vascular hypertrophy. Microvasc Res 59: 61-71, 2000.

12. Zufferey R, Nagy D, Mandel RJ, Naldini L and Trono D: Multiply attenuated lentiviral vector achieves efficient gene delivery in vivo. Nat Biotechnol 15: 871-875, 1997.

13. Huang W, Yan Z, Li D, Ma Y, Zhou J and Sui Z: Antioxidant and anti-inflammatory effects of blueberry anthocyanins on high glucose-induced human retinal capillary endothelial cells. Oxid Med Cell Longev 2018: 1862462, 2018.

14. Farjo KM, Farjo RA, Halsey S, Moiseyev G and Ma JX: Retinol-binding protein 4 induces inflammation in human endothelial cells by an NADPH oxidase- and nuclear factor kappa B-dependent and retinol-independent mechanism. Mol Cell Biol 32: 5103-5115, 2012

15. Francescone RA III, Faibish M and Shao R: A Matrigel-based tube formation assay to assess the vasculogenic activity of tumor cells. J Vis Exp, 2011.
16. Landt S, Heidecke H, Korlach S, Reuter C, Schwidde I, Barinoff J, Thill M, Sehouli J and Kümmel S: In vitro vascular tube formation testing as a tool for treatment individualisation in patients with cervical cancer. Anticancer Res 31: 2609-2615, 2011.

17. Livak KJ and Schmittgen TD: Analysis of relative gene expression data using real-time quantitative PCR and the 2(-Delta Delta C(T)) method. Methods 25: 402-408, 2001

18. Chlenski A, Dobratic M, Salwen HR, Applebaum M, Guerrero LJ, Miller R, DeWane G, Solomaha E, Marks JD and Cohn SL: Secreted protein acidic and rich in cysteine (SPARC) induces lipotoxicity in neuroblastoma by regulating transport of albumin complexed with fatty acids. Oncotarget 7: 77696-77706, 2016.

19. Kao SC, Kirschner MB, Cooper WA, Tran T, Burgers S, Wright C, Korse T, van den Broek D, Edelman J, Vallely M, et al: A proteomics-based approach identifies secreted protein acidic and rich in cysteine as a prognostic biomarker in malignant pleural mesothelioma. Br J Cancer 114: 524-531, 2016.

20. Abe T, Takahashi S and Suzuki N: Oxidative metabolism in cultured rat astroglia: Effects of reducing the glucose concentration in the culture medium and of D-aspartate or potassium stimulation. J Cereb Blood Flow Metab 26: 153-160, 2006.

21. Norose K, Lo WK, Clark JI, Sage EH and Howe CC: Lenses of SPARC-null mice exhibit an abnormal cell surface-basement membrane interface. Exp Eye Res 71: 295-307, 2000.

22. Bornstein P: Diversity of function is inherent in matricellular proteins: An appraisal of thrombospondin 1. J Cell Biol 130: 503-506, 1995 .

23. Bailey-Serres J, Sorenson R and Juntawong P: Getting the message across: Cytoplasmic ribonucleoprotein complexes. Trends Plant Sci 14: 443-453, 2009.

24. Watanabe K, Okamoto F, Yokoo T, Iida KT, Suzuki H, Shimano H, Oshika T, Yamada N and Toyoshima H: SPARC is a major secretory gene expressed and involved in the development of proliferative diabetic retinopathy. $\mathrm{J}$ Atheroscler Thromb 16: 69-76, 2009.

25. Song SJ, Han K, Choi KS, Ko SH, Rhee EJ, Park CY, Park JY, Lee KU and Ko KS; Task Force Team for Diabetes Fact Sheet of the Korean Diabetes Association: Trends in diabetic retinopathy and related medical practices among type 2 diabetes patients: Results from the National Insurance Service Survey 2006-2013. J Diabetes Investig 9: 173-178, 2017.

26. Wang FH, Sun XD, Zhang X, Xu X, Zhu Q, Huang JN, Fan Y, $\mathrm{Gu} \mathrm{Q}$ and Liu HY: Role of pigment epithelium-derived factor on proliferation and migration of choroidal capillary endothelium induced by vascular endothelial growth factor in vitro. Chin Med J (Engl) 120: 1534-1538, 2007.

27. Wang X, Wang G and Wang Y: Intravitreous vascular endothelial growth factor and hypoxia-inducible factor $1 \mathrm{a}$ in patients with proliferative diabetic retinopathy. Am J Ophthalmol 148: 883-889, 2009

28. Rubsam A, Parikh S and Fort PE: Role of inflammation in diabetic retinopathy. Int J Mol Sci 19: pii: E942, 2018.

29. Rivera LB, Bradshaw AD and Brekken RA: The regulatory function of SPARC in vascular biology. Cell Mol Life Sci 68: 3165-3173, 2011

30. Zhang JL, Chen GW, Liu YC, Wang PY, Wang X, Wan YL, Zhu J, Gao HQ, Yin J, Wang W and Tian ML: Secreted protein acidic and rich in cysteine (SPARC) suppresses angiogenesis by down-regulating the expression of VEGF and MMP-7 in gastric cancer. PLoS One 7: e44618, 2012.

31. Boyineni J, Tanpure S, Gnanamony M, Antony R, Fernández KS Lin J, Pinson D and Gondi CS: SPARC overexpression combined with radiation retards angiogenesis by suppressing VEGF-A via miR410 in human neuroblastoma cells. Int J Oncol 49: 1394-1406, 2016.

32. Rahimi N: Defenders and challengers of endothelial barrier function. Front Immunol 8: 1847, 2018.

33. Klaassen I, Van Noorden CJ and Schlingemann RO: Molecular basis of the inner blood-retinal barrier and its breakdown in diabetic macular edema and other pathological conditions. Prog Retin Eye Res 34: 19-48, 2013

34. Tan GS, Cheung N, Simo R, Cheung GC and Wong TY: Diabetic macular oedema. Lancet Diabetes Endocrinol 5: 143-155, 2017.

This work is licensed under a Creative Commons Attribution-NonCommercial-NoDerivatives 4.0 International (CC BY-NC-ND 4.0) License. 